\title{
Acta Epileptologica: for sharing research advances in epilepsy research
}

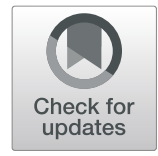

\author{
Shichuo Li(D)
}

Why are we building a new journal for epilepsy research? It's a question we kept thinking during the planning and preparation of Acta Epileptologica.

First, we are faced with greater challenges caused by Epilepsy. An aging society with more various public health problems has come, among which epilepsy is one of the arising risks for health. The $\mathrm{WHO}$ has estimated that epilepsy affects more than 50 million people worldwide and one of the peak age of onset is over 65 years old. Therefore, more research on finding solutions is urgently needed. Acta Epileptologica (AE) offers a new option for publication and communication.

Second, rapid advances in neurosciences and biotechnology have substantially transformed the research field of epileptology during past two decades, and even faster with the development of artificial intelligence and big data analyses. Therefore, Information should be exchanged more quickly. $\mathrm{AE}$ serves the community with rapid review and decision making.

Third, advances in genetic technology have given birth to precision medicine. In addition to the guidelines, there are more personalized options for epilepsy treatment. AE publishes interesting case reports in addition to research articles and reviews.

Moreover, while experts are calling for individualized treatment of epilepsy in most High income countries (HIC), doctors in rural areas and Low-and-middle Income Countries (LMIC) are still lacking the knowledge on basic principles of epilepsy treatment. Prompt access upon online of $\mathrm{AE}$ publications is worldwide and free. Therefore $\mathrm{AE}$ motivated to disseminate the evidence-based knowledge on epilepsy diagnosis and treatment worldwide. This would be an efficient educational approach to standardizing the treatment of epilepsy and finally reducing the epilepsy treatment gap.

For all of these reasons, the China Association Against Epilepsy (CAAE), with Sichuan University (SCU), in collaboration with the leading Open Access publishing brand

Correspondence: shichuoli@163.com

Acta Epileptologica, China Association Against Epilepsy, Beijing, China
BMC (part of Springer Nature), launches the journal Acta Epileptologica. For details of the Aims and Scope and the Submission guidelines of the journal, please check with our website: https://aepi.biomedcentral.com/.

We are publishing review and full research articles to mark the launch of Acta Epileptologica.

In the review titled "Strategies for Epilepsy Control in Public Health Aspects", Li et al. have summarized the related actions worldwide and some recommendations on the strategies for epilepsy prevention \& control in public health aspects. According to the whole advancement in the diagnosis, treatment and research of epilepsy has greatly brightened the therapeutic outlook for people with epilepsy. Of equal significance has been the increasing realization of the importance of preventive and community aspects of epilepsy, especially in some regions and countries. For further developing strategies for epilepsy control in public health aspects, authors recommend to the International League Against Epilepsy (ILAE), International Bureau for Epilepsy (IBE), state governments and other international \& regional organizations to amend and perfect the goal and mission of epilepsy control in three levels: Reference to policies and regulations by WHO; Public health strategies/actions in political and geographical aspects; Public health strategies/actions in technological field. This might be the admissible promotion and suggestions for decision makers and health workers.

In the research article Systemic evidence of acute seizure-associated elevation in serum neuronal injury biomarker in patients with temporal lobe epilepsy, Kunda et al. report that post-ictal assessment of serum neuron-specific enolase (NSE) may serve as a neural injury biomarker for measuring the efficacy of neural injury in temporal lobe epilepsy patients. Kunda and colleagues have measured serum levels of NSE, relative to levels of S100ßat 6 AM, 9 AM, noon, $3 \mathrm{PM}$, and $6 \mathrm{PM}$ over the course of several days in 7 epilepsy patients undergoing continuous video-EEG monitoring and in 4 healthy controls. They have found that NSE levels were significantly elevated after seizures or increased EEG spike frequency. They have presented that serum NSE may serve as a 
biomarker for measuring the efficacy of future acute neuroprotective strategies in epilepsy patients.

We hope you enjoy reading the first series of papers, and cordially invite you to share your experiences, discoveries, and insights with other readers on epilepsy research. Even more importantly, we look forward to your submissions to show your excellent research and opinion.

\section{Acknowledgements}

Not applicable.

Authors' contributions

The author Shichuo Li wrote the editorial.

\section{Funding}

Not applicable.

Availability of data and materials

Not applicable, no data concerned.

Ethics approval and consent to participate

Not applicable, no research conducted.

\section{Consent for publication}

Not applicable, no research conducted.

\section{Competing interests}

The author declare there're no competing interest.

Received: 28 June 2019 Accepted: 28 June 2019 Published online: 25 July 2019 